\title{
Avaliação dos parâmetros nutricionais e metabólicos de borregas alimentadas com leveduras na ração
}

\author{
Marco Túlio Santos Siqueira ${ }^{1 *}$; Paulo Arthur Cardoso Ruela²; Karla Alves Oliveira ${ }^{3}$; Débora Adriana de Paula \\ Silva $^{4}$; Luciano Fernandes Sousa ${ }^{5}$; Gilberto de Lima Macedo Júnior ${ }^{6}$
}

DOI: https://doi.org/10.35699/2447-6218.2020.23902

\begin{abstract}
Resumo
Objetivou-se avaliar a digestibilidade da matéria seca, consumo e perfil metabólico de borregas alimentadas com leveduras vivas e inativadas na ração. Foram utilizados vinte animais mestiços com peso médio de $37,3 \mathrm{~kg}$. Os alimentos foram fornecidos duas vezes ao dia e pesados e amostrados diariamente, também foram amostradas as sobras e as fezes na matéria natural. Foi mensurada a quantidade de urina excretada bem como sua densidade. Todas as coletas sanguíneas foram realizadas de manhã com animais em jejum. Utilizou-se o delineamento inteiramente casualizado, com quatro tratamentos e cinco repetições. Foram submetidos à análise variância e as médias dos tratamentos comparadas pelo teste SNK com nível de significância de 5\% para o erro tipo I. Foi considerada tendência significativa o P valor maior que 0,05 e menor que 0,10. Avaliou-se os seguintes tratamentos: Milk Sacc X ${ }^{\circledR}$ (levedura ativa), Active Flora ${ }^{\circledR}$ (levedura viva mais levedura inativada) e Rúmen Yeast ${ }^{\circledR}$ (levedura inativa). Não houve diferença entre tratamentos para CMS e DMS, assim como para as demais variáveis analisadas com exceção do escore fecal, que apresentou tendência significativa $(\mathrm{P}=0,0682)$, cujos tratamentos Active Flora ${ }^{\circledR}$ e Rúmen Yeast ${ }^{\circledR}$ foram ideais para a espécie. Nenhum tratamento apresentou diferença estatística para as variáveis metabólicos sanguíneos. Porém, houve efeito quadrático para horário de avaliação para a concentração de glicose sanguínea. A inclusão de leveduras vivas ou inativadas na dieta para borregas não influencia na digestibilidade da dieta, no consumo de matéria seca dos animais e também não altera o perfil metabólico dos mesmos.
\end{abstract}

Palavras-chave: Aditivo microbiano. Metabólitos. Ovis aries. Ruminantes.

\section{Evaluation of nutritional and metabolic parameters of lambs fed with yeast in the feed}

\author{
Abstract \\ ${ }^{1}$ Universidade Federal de Uberlândia. Uberlândia, MG. Brasil. \\ https://orcid.org/0000-0002-2098-8568 \\ ${ }^{2}$ Universidade Federal de Uberlândia. Uberlândia, MG. Brasil. \\ https://orcid.org/0000-0001-9136-3920 \\ 3Universidade Estadual Paulista "Júlio de Mesquita Filho". Jaboticabal, SP. Brasil. \\ https://orcid.org/0000-0002-7792-2615 \\ ${ }^{4}$ Universidade Federal de Uberlândia. Uberlândia, MG. Brasil. \\ https://orcid.org/0000-0003-3052-0544 \\ ${ }^{5}$ Universidade Federal de Tocantins. Araguaína, TO. Brasil. \\ https://orcid.org/0000-0002-6072-9237 \\ ${ }^{6}$ Universidade Federal de Uberlândia. Uberlandia, MG. Brasil. \\ https://orcid.org/0000-0001-5781-7917 \\ *Autor para correspondência: marcotulio.s.siqueira@gmail.com
}

The objective was to evaluate dry matter digestibility, consumption and metabolic profile of lambs fed with live and inactivated yeasts in the feed. Twenty crossbred animals with an average weight of $37.3 \mathrm{~kg}$ were used. The food was supplied twice a day and weighed and sampled daily, the leftovers and feces in natural matter were also sampled. The amount of urine excreted and its density were measured. All blood collections were performed in the morning

Recebido para publicação em 01 de Julho de 2020. Aceito para publicação 25 de Novembro de 2020

e-ISSN: 2447-6218 / ISSN: 2447-6218. Atribuição CC BY. 
with fasting animals. A completely randomized design was used, with four treatments and five replications. Variance analysis and treatment averages were compared using the SNK test with a 5\% significance level for type I error. P value greater than 0.05 and less than 0.10 was considered a significant trend. The following treatments were evaluated: Milk Sacc $\mathrm{X}^{\circledR}$ (active yeast), Active Flora ${ }^{\circledR}$ (live yeast plus inactivated yeast) and Rumen Yeast ${ }^{\circledR}$ (inactive yeast). There was no difference between treatments for CMS and DMS, as well as for the other variables analyzed with the exception of the fecal score, which showed a significant trend $(\mathrm{P}=0.0682)$, whose Active Flora ${ }^{\circledR}$ and Rumen Yeast ${ }^{\circledR}$ treatments were ideal for the species. No treatment showed a statistical difference for blood metabolic variables. However, there was a quadratic effect on the time of assessment for blood glucose concentration. The inclusion of live or inactivated yeasts in the diet for lambs does not influence the digestibility of the diet, the consumption of dry matter of the animals and also does not change their metabolic profile.

Keywords: Digestibility. Microbial Additive. Ovis aries. Ruminants.

\section{Introdução}

Leveduras são fungos unicelulares e o principal gênero na indústria alimentícia é o Saccharomyces, que possui grande importância na nutrição animal. O uso de leveduras na alimentação de ruminantes tem como finalidade regular mudanças no balanço e atividade das comunidades de microrganismos no rúmen, associadas a desordens e distúrbios metabólicos, tais como cetose e timpanismo. Essas alterações metabólicas promovem queda no desempenho produtivo, além de aumentar o risco à saúde dos animais, uma vez que limitam consumo e debilitam os animais. Assim, as leveduras como probióticos auxiliam na estabilidade da microbiota evitando desequilíbrio no ecossistema ruminal (Neumann et al., 2008).

Ainda segundo Neumann et al. (2008) as leveduras podem ser disponibilizadas na forma ativa e na forma inativa. Tem-se que a forma ativa (viva) apresenta melhores benefícios na alimentação de ruminantes, controlando as concentrações de ácido lático no rúmen, fornecendo fatores estimulatórios às bactérias do mesmo e absorvendo oxigênio no ambiente ruminal. Já as leveduras na forma inativa atuam na manutenção do $\mathrm{pH}$ e estimulação da atividade das bactérias celulolíticas, proporcionando uma melhor condição do rúmen.

Diversos são os trabalhos publicados que avaliam a inclusão de leveduras na dieta de ovinos, no entanto resultados são focados em consumo e digestibilidade, não sendo verificados os metabólitos sanguíneos dos animais. Aguiar et al. (2007) avaliaram o efeito da substituição do milho e farelo de soja por levedura de cana de açúcar e ureia, sobre o consumo e digestibilidade de nutrientes e desempenho, em ovinos. E relataram que tal dieta afetou negativamente o consumo de energia e o desempenho animal. Tavares et al. (2020) avaliaram efeitos da suplementação com levedura Saccharomyces cerervisiae sobre o consumo de matéria seca e ganho médio diário em ovinos que passaram por trocas bruscas de dieta na relação volumoso:concentrado. Os autores observaram que tanto a levedura ativa, quanto autolisada melhoraram o desempenho animal, aumentando o peso dos mesmos.
A ação sinérgica desses aditivos incluídos nas dietas contribui para melhores condições no ambiente ruminal, de modo que as enzimas atuam no melhor aproveitamento dos nutrientes, melhorando a digestibilidade da matéria seca. Por outro lado, as leveduras ampliam as condições do rúmen gerando um ambiente que efetiva a ação dos microrganismos ruminais. As enzimas usadas são importantes em dietas de alta proporção de grãos como a deste presente estudo. Tal fato já é conhecido na literatura, como demonstra Igarasi et al. (2008), onde destaca que rações contendo altos níveis de grãos possibilitam maior desempenho aos animais, entretanto mesmo que moído ou farelado, esse ingrediente é protegido pelo pericarpo, estrutura resistente à degradação microbiana e à digestão enzimática.

Diante disso, acredita-se que a inclusão de leveduras ativas e inativadas na ração de borregas contendo enzimas possa aumentar a digestibilidade da matéria seca e consumo pelos animais sem prejuízos metabólicos aos mesmos. Assim, tem-se por objetivo neste estudo avaliar os efeitos da inclusão de leveduras vivas, inativadas e vivas mais inativadas na ração para borregas contendo mix de enzimas, sobre como afetam o consumo de matéria seca, digestibilidade dos alimentos e metabólitos sanguíneos.

\section{Material e métodos}

O experimento foi conduzido na Universidade Federal de Uberlândia, setor de pequenos ruminantes, em Uberlândia, Oeste de Minas Gerais, Brasil, que apresentou clima com temperatura e umidade relativa do ar médias de $23,6^{\circ} \mathrm{C}$ e $80,7 \%$, respectivamente durante a experimentação segundo dados do CLIMA (Laboratório de climatologia e Meteorologia Ambiental).

Foram utilizadas 20 borregas (cada animal constituiu uma unidade experimental), Dorper x Santa Inês com média inicial de $37,3 \mathrm{~kg}$ de peso corporal (PC) e sete meses de idade. Os animais foram mantidos em gaiolas de metabolismo, providas de bebedouro, cocho e saleiro, conforme padrão dos Institutos Nacionais de Ciência e Tecnologia (INCT). Foram pesadas, identificadas, vermifugadas com Zolvix ${ }^{\circledR}$ (Novartis Saúde Animal, 
Avaliação dos parâmetros nutricionais e metabólicos de borregas alimentadas com leveduras na ração

Basileia-Cidade, Basileia, França) na dose de 2,5 mg de Monepantel por $\mathrm{kg}$ de peso vivo, vacinadas contra raiva, leptospirose, clostridioses e botulismo; e sorteadas entre os grupos experimentais. O ensaio teve duração de 20 dias, no mês de janeiro de 2019, sendo 15 dias de adaptação e cinco dias de coleta de dados e amostragens.

Foram testados quatro tratamentos: controle (sem adição de leveduras), Milk Sacc $\mathrm{X}^{\circledR}$ ((Alltech ${ }^{\circledR}$, Maringá, Paraná, Brasil), levedura ativa - Saccharomyces cerevisiae cepa $1026,5,0 \times 10^{8} \mathrm{UFC} \mathrm{g}^{-1}$ ) na dose de 0,0015kg animal dia-1 ${ }^{-1}$ Rúmen Yeast ${ }^{\circledR}$ ((York Ag Products INC., York, Pensilvânia, Estados Unidos), levedura inativa - Saccha- romyces cerevisiae, com $1,5 \times 10^{4} \mathrm{UFC}^{-1}$ ) na dose de $0,0045 \mathrm{~kg}$ animal dia $^{-1}$ e Active Flora ${ }^{\circledR}$ ((ICC, Louisville, Kentucky, Estados Unidos), levedura viva mais levedura inativada - Saccharomyces cerevisiae, com 2,0 x $10^{10}$ UFC $\mathrm{g}^{-1}$ ) na dose de $0,003 \mathrm{~kg}$ animal dia $^{-1}$. As alimentações ocorreram às 8:00 e 16:00 horas, sendo ofertados 50\% do total em cada alimentação.

A dieta experimental foi composta por silagem de milho (30,0\%) e concentrado (70,0\%) e balanceada segundo o NRC (2007) para ganhos de $300 \mathrm{~g} \mathrm{dia}^{-1}$, de forma que houvessem sobras entre $5-10 \%$ do total fornecido (Tabela 1 ).

Tabela 1 - Composição percentual e bromatológica (\%) do concentrado e silagem de milho

\begin{tabular}{lcc}
\hline Composição das dietas/tratamentos & Concentrado & Silagem de milho \\
\hline Milho farelo & 72,00 & - \\
Farelo de soja & 18,00 & - \\
Ureia & 2,00 & - \\
Sal mineral & 5,00 & - \\
Mix de enzimas** & 3,00 & - \\
Adsorvente & $200 g$ & 32,20 \\
\hline Matéria seca & - & 6,30 \\
Proteína bruta & - & 62,63 \\
Nitrogênio digestível total & - & 54,60 \\
Fibra em detergente neutro & - & 32,60
\end{tabular}

*Sem adição de leveduras; **Mix de enzimas contém: Amaize ${ }^{\mathrm{TM}}$ (extrato de Aspergillus oryzae); Allzyme ${ }^{\circledR}$ (Aspergillus niger) e Fibrozyme ${ }^{\circledR}$ (Trichoderma longibarachiatu). Informações obtidas pelas análises feitas no laboratório de nutrição animal da UFU.

Na tabela 2 estão os valores da composição das enzimas utilizadas no estudo como ingrediente dos concentrados utilizados na ração experimental. Para confecção do mix de enzima foram utilizados $75 \mathrm{~g}$ de Amaize $^{\mathrm{TM}}$, $75 \mathrm{~g}$ de Allzyme ${ }^{\circledR}$ e $90 \mathrm{~g}$ de Fibrozyme, totalizando $240 \mathrm{~g}$ de mix de enzimas (produto). Esse produto foi adicionado aos ingredientes do concentrado (conforme proporções na tabela 1) no misturador vertical de $200 \mathrm{~kg}$, misturando por 15 minutos.

Tabela 2 - Composição das enzimas utilizadas como ingrediente do concentrado de acordo o fabricante

\begin{tabular}{|c|c|c|c|}
\hline Composição & Allzyme $^{\circledR}$ & Amaize $^{\mathrm{TM}}$ & Fibrozyme $^{\circledR}$ \\
\hline Pectinase & Min. $400 \mathrm{u}^{*} \mathrm{~g}^{-1}$ & - & - \\
\hline Protease & Min. $700 \mathrm{u}^{*} \mathrm{~g}^{-1}$ & - & - \\
\hline Fitase & Min. $300 \mathrm{u}^{*} \mathrm{~g}^{-1}$ & - & - \\
\hline Betaglucanase & Min. $200 \mathrm{u}^{*} \mathrm{~g}^{-1}$ & - & - \\
\hline Xilanase & Min. $100 \mathrm{u}^{*} \mathrm{~g}^{-1}$ & - & Min. $100 \mathrm{XU}^{* * *} \mathrm{~g}^{-1}$ \\
\hline Celulase & Min. $40 \mathrm{u}^{*} \mathrm{~g}^{-1}$ & - & - \\
\hline Amilase & Min. $30 \mathrm{u}^{*} \mathrm{~g}^{-1}$ & Min. 600 FAU $^{* *} \mathrm{~g}^{-1}$ & - \\
\hline
\end{tabular}


$\mathrm{pH} 4,8$ e $30^{\circ} \mathrm{C} ; * * *$ Uma unidade de atividade enzimática xilanase equivalente à quantidade de enzima que libera 1 micromol de xilose por minuto a partir de xilano a pH 5,3 e $50^{\circ} \mathrm{C}$.

Durante o período de coleta foram pesados e amostrados diariamente o alimento oferecido, sobras e fezes na matéria natural em balança com precisão de 5 g. Foi feita uma amostra composta a partir das amostras simples, para cada animal durante os cinco dias de coleta. A urina era acondicionada em baldes adaptados com tela para separação das fezes que eram recolhidas em bandejas plásticas. $\mathrm{O}$ volume total de urina foi medido em proveta de plástico de $2 \mathrm{~L}$ com precisão de $20 \mathrm{~mL}$ e a densidade da urina foi determina em refratômetro manual portátil da Megabrix ${ }^{\circledR}$ (Fremont, Ohio, Estados Unidos).

Foi avaliado também o escore fecal, de acordo com escala proposta por Gomes et al. (2012), sendo que na escala um (1) as fezes são ressecadas e sem brilho; na escala dois (2) as fezes são normais; na escala três (3) as fezes são ligeiramente amolecidas; na escala quatro (4) as fezes são amolecidas, perdendo o formato e coladas umas às outras (cacho de uva); na escala cinco (5) as fezes são amolecidas e sem formato normal (fezes de suínos); e na escala seis (6) as fezes são diarreicas.

As amostras de alimentos, sobras e fezes foram acondicionadas em sacos plásticos, identificadas e armazenadas em congelador a $-15^{\circ} \mathrm{C}$. Ao final do ensaio, as amostras foram descongeladas e homogeneizadas, sendo retirada amostra de $20 \%$ do total para posteriores análises laboratoriais. Os teores de matéria seca foram obtidos pelo método INCT-CA G-003/1. Posteriormente calculou-se a matéria seca definitiva e, a digestibilidade aparente da dieta conforme as equações (Eq. 1 e 2) propostas por Maynard et al. (1984):

$$
\begin{gathered}
\text { (Eq. 1) } C N=(\text { Cons } X \% \text { Cons })-(\text { Sob X\%Sob }) \\
\text { (Eq. 2) } D A=\frac{C N-(\text { Fez X \% Fez })}{C N} X 100
\end{gathered}
$$

Sendo $\mathrm{CN}=$ consumo do nutriente $(\mathrm{kg}) ;$ Cons $=$ quantidade de alimento consumido $(\mathrm{kg}) ; \%$ cons = teor do nutriente no alimento fornecido (\%); Sob = quantidade de sobra retirada $(\mathrm{kg}) ; \%$ sob $=$ teor do nutriente nas sobras (\%); DA = digestibilidade aparente (\%); Fez $=$ quantidade de fezes coletada $(\mathrm{kg}) ; \% \mathrm{fez}=$ teor do nutriente nas fezes (\%).

O cálculo do consumo de água de bebida foi baseado na diferença entre o ofertado e as sobras, subtraindo o valor evaporado. Todos os dias foram ofertados para cada animal uma quantidade padrão de seis litros de água, sendo acrescida maior quantidade quando necessário. Foi utilizado um balde de água para controle da evaporação a cada 24 horas, sendo adicionados seis litros de água e no dia seguinte contabilizado a sobra. A quantidade de água evaporada foi descontada do consumo de água de cada animal.

As amostras de sangue foram coletadas por venopunção da jugular com auxílio de tubos Vacutainer ${ }^{\circledR}$ (BD, São Paulo, São Paulo, Brasil) de cinco mL contendo fluoreto e EDTA, sendo devidamente identificados para cada animal. A avaliação da curva glicêmica foi realizada no último dia de coleta, já para os componentes bioquímicos foram realizadas no primeiro, terceiro e quinto dia de coleta do experimento. Essas colheitas ocorreram antes da primeira alimentação com o animal em jejum. Para a avaliação glicêmica as colheitas foram feitas às $8: 00 \mathrm{~h}$ (antes da primeira refeição), 11:00h, 14:00h, 17:00h e às $20: 00 \mathrm{~h}$. No dia da avaliação glicêmica a segunda refeição somente foi ofertada após a colheita das 20:00h. Não foram mensurados os efeitos do estresse da coleta a cada três horas nos animais.
As amostras de sangue coletadas foram centrifugadas a 3000 rotações por minuto por 10 minutos, sendo os soros separados em alíquotas, guardados em microtubos e armazenados em freezer a $-5^{\circ} \mathrm{C}$ para posterior análise laboratorial. Todas as amostras foram processadas no analisador bioquímico automatizado Bioplus ${ }^{\circledR} 2000$ (Bioplus Produtos para Laboratório Ltda., Barueri, São Paulo, Brasil), usando kit comercial da Lab Test Diagnóstica S. A. ${ }^{\circledR}$ (Lagoa Santa, Minas Gerais, Brasil).

Os componentes bioquímicos avaliados para determinação do metabolismo energético foram: triglicerídeos, colesterol, VLDL (lipoproteína de muito baixa densidade, calculado através da fórmula proposta por Friedewald, Levv e Fredrickson (1972): VLDL = triglicerídeos $\div 5$ ) e frutosamina; para determinar função hepática foram: gama glutamiltransferase (GGT), aspartato aminotransferase (AST) e fosfatase alcalina (FA); para determinação do metabolismo proteico foram: proteína total (PT), ureia, albumina, ácido úrico e creatinina.

O experimento foi realizado em delineamento inteiramente casualizado, com quatro tratamentos, sendo um grupo controle e três contendo diferentes formulações com leveduras. Cada tratamento dispôs de cinco repetições. Todos os dados foram testados quanto à normalidade (Shapiro e Wilk, 1965) e homocedasticidade (Levene, 1960) de variância do resíduo. Aceitos estes pressupostos os dados foram submetidos à análise variância e as médias dos tratamentos comparadas pelo teste SNK (Student-Newman-Keuls) com nível de significância de 5\% para o erro tipo I. A variável escore fecal por ser não paramétrica foi avaliada pelo teste de Kruskal e Wallis (1952) ao nível de significância de $5 \%$. A variável glicemia (horário de colheita) foi avaliada como parcela subdivida, onde nas parcelas tinham-se os tratamentos e 
Avaliação dos parâmetros nutricionais e metabólicos de borregas alimentadas com leveduras na ração

nas sub-parcelas os horários de colheita $(8 \mathrm{~h}, 11 \mathrm{~h}, 14 \mathrm{~h}$, $17 \mathrm{~h}$ e $20 \mathrm{~h}$ ). Foi considerada tendência significativa o $P$ valor maior que 0,05 e menor que 0,10 conforme se observa na discussão atual dos trabalhos internacionais publicados em revistas indexadas.

\section{Resultados e discussão}

Não houve diferença estatística $(P>0,05)$ entre os grupos avaliados para as variáveis consumo de matéria seca (CMS) em $\mathrm{kg} \mathrm{dia}^{-1}$, em porcentagem do peso corporal e peso metabólico, e para digestibilidade na matéria seca (DMS, Tabela 3). O CMS médio foi de 1,20 $\pm 0,18 \mathrm{~kg} \mathrm{dia}^{-1}$, se enquadrando dentro do recomendado para a categoria animal analisada que é de 1,0-1,3 kg dia $^{-1}$ segundo NRC (2007). Já o CMS em relação ao peso corporal (CMS\%PC) recomendado pelo NRC (2007) é de $3,51 \%$, logo os valores de CMS\%PC observados estiveram todos próximos do recomendado.

Tabela 3 - Consumo e digestibilidade da matéria seca em borregas alimentadas ou não com leveduras na dieta

\begin{tabular}{lcccc}
\hline Tratamento & CMS (kg dia-1) & CMS (\%PC) & CMS (PC $\left.{ }^{0,75}\right)$ & DMS (\%) \\
\hline Controle & 1,28 & 3,46 & 85,45 & 83,90 \\
Active Flora $^{\circledR}$ & 1,13 & 3,06 & 75,44 & 81,13 \\
Milk Sacc X $^{\circledR}$ & 1,33 & 3,90 & 94,26 & 84,51 \\
Rúmen Yeast ${ }^{\circledR}$ & 1,13 & 3,02 & 74,84 & 79,05 \\
P Valor & 0,4741 & 0,3000 & 0,2714 & 0,2162 \\
MG & 1,20 & 3,29 & 80,94 & 81,77 \\
CV & 17,69 & 20,42 & 18,21 & 4,84 \\
\hline
\end{tabular}

MG: média geral; CV: coeficiente de variação (\%).

Os alimentos utilizados na dieta (farelo de soja e milho, silagem e ureia; Tabela 1) serviram como fonte de nitrogênio degradável no rúmen (PDR), maximizando a síntese de proteína microbiana, uma vez que esta é dependente da concentração e qualidade das fontes de energia (especialmente amido) no rúmen (Cruz et al. 2010). Esse equilíbrio entre as fontes pode ter contribuído em manter o consumo de alimento estável. Além disso, todas as dietas eram iguais, possuíam um mix de enzimas exógenas, alterando apenas o tipo de levedura, o que possivelmente contribuiu para esse resultado, tornando a microbiota ruminal estável.

A média da DMS do presente estudo foi de $81,77 \%$, considerada alta digestibilidade da dieta. O CMS esteve dentro do recomendado pela literatura, e pode-se inferir que houve bom aproveitamento dos alimentos pelos animais, uma vez que ocorreu alta digestibilidade da matéria seca sem redução do consumo. Logo, pode-se inferir que o valor encontrado para a digestibilidade neste estudo deveu-se ao efeito associativo das leveduras e do mix de enzimas presente no concentrado. As enzimas utilizadas neste estudo têm por objetivo aumentar a degradação das frações indigestíveis de alguns alimentos, com sinergismo as enzimas endógenas do rúmen. Elas são essenciais, pois estão envolvidas na hidrólise de alimentos complexos em suas moléculas orgânicas mais simples, como glicose, celobiose, xilose, aminoácidos e ácidos graxos, que então são utilizados pelos microrganismos do rúmen (Kozloski, 2011). Aguiar et al. (2007) ao avaliar com níveis crescentes de levedura na dieta em substituição ao farelo de milho e farelo de soja sem adição de enzimas encontrou valores de DMS de 70,04\%, $66,33 \%, 66,21 \%$ e $62,76 \%$.

Não se observou influência $(\mathrm{P}>0,05)$ das dietas avaliadas para consumo de água $\left(\mathrm{CH}_{2} \mathrm{O}\right)$, volume de urina, densidade de urina, massa de fezes na matéria natural (MFMN) e fezes na matéria seca (FMS). Houve tendência significativa para a variável escore fecal $(\mathrm{P}=0,0682)$, onde os tratamentos Active Flora ${ }^{\circledR}$ e Rúmen Yeast ${ }^{\circledR}$ se mostraram superiores aos demais tendo em vista o método de avaliação em graus de escore fecal sugerido por Gomes et al. (2012), ou seja, ambas obtiveram o valor recomendado para a espécie ovina (escore 2) (Tabela 4). As leveduras são responsáveis por modular a fermentação ruminal, alterando a configuração da microbiota do rúmen. melhorando a digestibilidade e consequentemente o aproveitamento do alimento, o que causa redução da excreção, com fezes mais consistentes (Gomes et al., 2012).

A média observada para $\mathrm{CH}_{2} \mathrm{O}$ dos animais foi de 3,64 litros de água por dia. Através da equação proposta pelo NRC (2007) que possibilita calcular a exigência para ingestão de água diária para ovelhas através do CMS: $\mathrm{CH}_{2} \mathrm{O}=3,86$ x CMS - 0,99. Utilizando a média do consumo de matéria seca (CMS) encontrado para todos os tratamentos $\left(1,20 \mathrm{Kg} \mathrm{dia}^{-1}\right)$, têm-se que a ingestão de água recomendada é de 3,64 litros por dia, ou seja, os animais ingeriram exatamente a quantidade de água recomendada. Foi estabelecida relação entre o consumo de água bebida e a quantidade de matéria seca ingerida para ovinos pelo NRC (2007), devendo ser duas a três 
Siqueira, M. T. S. et al.

vezes maior que o CMS, logo de acordo com esta reco-

mendação o $\mathrm{CH}_{2} \mathrm{O}$ dos animais neste experimento se encontra dentro da normalidade.

Tabela 4 - Consumo de água, parâmetros fecais e urinários de borregas alimentadas ou não com leveduras

\begin{tabular}{|c|c|c|c|c|}
\hline Tratamento & $\mathrm{CH}_{2} \mathrm{O}\left(\mathrm{L} \mathrm{dia}^{-1}\right)$ & $\begin{array}{l}\mathrm{CH}_{2} \mathrm{O} / \mathrm{CMS} \\
\left(\mathrm{L} \mathrm{Kg}^{-1} \mathrm{dia}^{-1}\right)\end{array}$ & VU $\left(\mathrm{L} \operatorname{dia}^{-1}\right)$ & $\mathrm{DU}\left(\mathrm{g} \mathrm{mL}^{-1}\right)$ \\
\hline Controle & 4,14 & 2,77 & 1,84 & 1,0204 \\
\hline Active Flora ${ }^{\circledR}$ & 3,03 & 2,94 & 1,66 & 1,0244 \\
\hline Milk Sacc $X^{\circledR}$ & 3,32 & 1,33 & 1,30 & 1,0248 \\
\hline Rúmen Yeast ${ }^{\circledR}$ & 3,82 & 3,34 & 1,33 & 1,0216 \\
\hline P Valor & 0,5394 & 0,1280 & 0,5091 & 0,7584 \\
\hline MG & 3,64 & 2,60 & 1,53 & 1,0228 \\
\hline $\mathrm{CV}$ & 28,89 & 30,99 & 32,65 & 0,75 \\
\hline Tratamento & MSF (\%) & FMS $\left(\mathrm{Kg} \mathrm{dia}^{-1}\right)$ & $\operatorname{MFMN}\left(\mathrm{Kg} \mathrm{dia}^{-1}\right)$ & Escore Fecal* \\
\hline Controle & 33,02 & 0,175 & 0,521 & $1,74 \mathrm{~B}$ \\
\hline Active Flora ${ }^{\circledR}$ & 30,45 & 0,190 & 0,575 & $2,24 \mathrm{~A}$ \\
\hline Milk Sacc $X^{\circledR}$ & 33,81 & 0,190 & 0,548 & $1,92 \mathrm{AB}$ \\
\hline Rúmen Yeast ${ }^{\circledR}$ & 31,31 & 0,194 & 0,572 & $2,24 \mathrm{~A}$ \\
\hline P Valor & 0,6509 & 0,9739 & 0,8522 & 0,0682 \\
\hline MG & 32,15 & 0,188 & 0,554 & 2,03 \\
\hline $\mathrm{CV}$ & 14,36 & 33,07 & 30,17 & $\mathrm{XXX}$ \\
\hline
\end{tabular}

*Estatística não paramétrica; MG: média geral; CV: coeficiente de variação; Letras distintas na coluna diferem-se pelo Teste de SNK à 5\% de significância.

Em relação ao volume urinário (VU) não houve diferença em função do tipo de levedura. A média geral da produção de urina apresentada foi de 1,53 $\mathrm{L} \mathrm{dia}^{-1}$. Para Reece (2006), em ovinos a excreção de urina deve ficar entre 0,1 e $0,4 \mathrm{~L}$ para cada $10 \mathrm{~kg}$ de peso vivo. Os animais em estudo tinham peso médio de $37,3 \mathrm{Kg}$, ou seja, a excreção de urina deve variar entre $0,4-1,5 \mathrm{~L}$ podendo afirmar, portanto que a excreção média de urina apresentada pelas borregas foi compatível com a faixa de recomendação, uma vez que o consumo de água também esteve dentro do recomendado.

Não houve diferença da densidade de urina (DSD) em função do tipo de levedura associado ao uso de enzimas exógenas para as borregas em estudo. $\mathrm{O}$ valor médio detectado para essa variável foi de 1,0228, considerada normal para a espécie. Segundo Carvalho (2008), para ovinos a variação da densidade urinária é entre 1,0150 e 1,0450. Avaliando os valores encontrados para $\mathrm{CH}_{2} \mathrm{O}$, $\mathrm{CH}_{2} \mathrm{O} / \mathrm{CMS}$, DSD e VU, pode-se afirmar que os animais não tiveram restrição hídrica.

Não foram observadas diferenças $(\mathrm{P}>0,05)$ para peso de fezes na matéria natural (MFMN), na matéria seca e matéria seca fecal (Tabela 4). O peso das fezes pode estar relacionado com a composição da dieta, taxa de passagem do alimento pelo rúmen e sua digestibilidade
(Santos e Nogueira, 2012). Como as dietas continham a mesma relação volumoso:concentrado, não houve efeito no uso de leveduras no peso das fezes e na digestibilidade (Tabela 3). De acordo com Vieira (2008) uma ovelha adulta produz entre 0,8 e 1,5 kg de fezes dia $^{-1}$ em matéria natural. Logo os animais em questão tiveram produção de $0,3 \mathrm{~kg} \mathrm{dia}^{-1}$ menor que o recomendado, uma vez que se tratavam de animais em crescimento. Segundo Van Cleef et. al., (2010), os valores de referência para matéria seca fecal (FMS) para a espécie ovina variam de $37 \%$ a $44 \%$. Sendo assim, essa se manteve abaixo dos valores mencionados $(32,15 \%)$.

Neste estudo não foi observada diferença $(P>0,05)$ entre os tratamentos para as concentrações dos metabólitos energéticos (Tabela 5). A verificação dos metabólitos sanguíneos é uma ferramenta utilizada para melhor interpretar os resultados de CMS e DMS, de modo que se utiliza esta mensuração para observar se houve algum tipo de deficiência nutricional ou sobrecarga hepática e renal durante os processos de metabolização.

Não houve diferença estatística entre os tratamentos para o metabólito triglicerídeos, de modo que esse encontra-se dentro da faixa de referência citada por Silva et al. (2020). A VLDL é responsável pelo transporte de triglicerídeos, logo, é esperado que apresentem 
comportamentos semelhantes (Santos et al., 2015). Essa variável permaneceu dentro da faixa de referência em todos grupos avaliados, o que indica adequada capacidade de transporte de moléculas de triglicerídeos ao longo do período experimental. Os teores de colesterol também estiveram dentro do esperado para todos os grupos avaliados. Villa et al. (2009) afirmam que os níveis de colesterol total plasmático são indicadores adequados do total de lipídeos no plasma, e tem uma relação direta com a alimentação do animal, o que mostra que a dieta foi eficiente em manter o metabolismo energético dos animais.

Tabela 5 - Concentração média dos metabólitos energéticos de borregas alimentadas ou não com leveduras na dieta

\begin{tabular}{lccccc}
\hline Tratamento & $\begin{array}{c}\text { Triglicerídeos } \\
(\mathbf{m g ~ d L})^{-1}\end{array}$ & $\begin{array}{c}\text { VLDL } \\
\left(\mathbf{g ~ d L}^{-1}\right)\end{array}$ & $\begin{array}{c}\text { Colesterol } \\
\left(\mathbf{m g ~ d L}^{-1}\right)\end{array}$ & $\begin{array}{c}\text { Frutosamina } \\
\left(\mu \mathbf{m o l ~ L}^{-1}\right)\end{array}$ & $\begin{array}{c}\text { Glicemia } \\
\left(\mathbf{m g ~ d L}^{-1}\right)\end{array}$ \\
\hline Controle & 36,26 & 7,25 & 46,80 & 146,10 & 62,24 \\
Rúmen Yeast $^{\circledR}$ & 36,46 & 7,29 & 53,86 & 148,50 & 62,96 \\
Active Flora $^{\circledR}$ & 38,26 & 7,65 & 54,66 & 157,50 & 66,48 \\
Milk Sacc X $^{\circledR}$ & 40,33 & 8,06 & 54,60 & 159,50 & 59,96 \\
P Valor & 0,9655 & 0,9654 & 0,6691 & 0,3943 & 0,6042 \\
MG & 37,83 & 7,56 & 52,48 & 154,90 & 62,91 \\
CV & 37,64 & 37,65 & 22,31 & 9,37 & 18,17 \\
VR* & $5-71$ & $1-16,40$ & $14-126,00$ & $119,00-451,00$ & $30,0-94,0$ \\
\hline
\end{tabular}

MG: média geral; CV: coeficiente de variação; VR*: valor de referência para ovinos segundo Silva et al. (2020).

A frutosamina é uma cetoamina estável e formada quando a glicose reage não enzimaticamente com grupos aminas das proteínas, principalmente a albumina e a imunoglobulina e sua concentração no plasma ou sérica é controlada pelo balanço entre a síntese e eliminação destes compostos proteicos e de glicose (Gouveia et al., 2015). Não houve diferença $(P>0,05)$ para esta variável, com todos os tratamentos permanecendo dentro da faixa recomendada por Silva et al. (2020).

Não houve diferença entre os tratamentos para a variável glicose (Tabela 5). Contudo, houve efeito quadrático para horário de avaliação para a concentração da mesma (Gráfico1). A glicose plasmática em animais ruminantes possui como precursor compostos que não são carboidratos, como o ácido graxo volátil (AGV) propionato. Após a absorção pelo epitélio ruminal, o propionato segue via corrente sanguínea para o fígado, sendo convertido em glicose na gliconeogênese para enfim ser usado como fonte de energia pelo animal (Kaneko et al., 2008). Portanto, dietas com relação de concentrado maior que a de volumosos, como a deste estudo, permitem maior incremento de ácido propiônico, que é o único dos AGV's precursor de glicose no ruminante, a partir da fermentação dos carboidratos solúveis, consequentemente aumentando a concentração de glicose no plasma. Por ser uma via indireta, seu tempo de produção é relativamente demorado, o que explica as 6 h necessárias para aumento da glicose no sangue, com o pico acontecendo 9h após a ingestão.

Gráfico 1 - Concentração de glicose $\left(\mathrm{mg} \mathrm{dL}^{-1}\right)$ em relação ao horário de coleta em borregas alimentadas ou não com leveduras na dieta

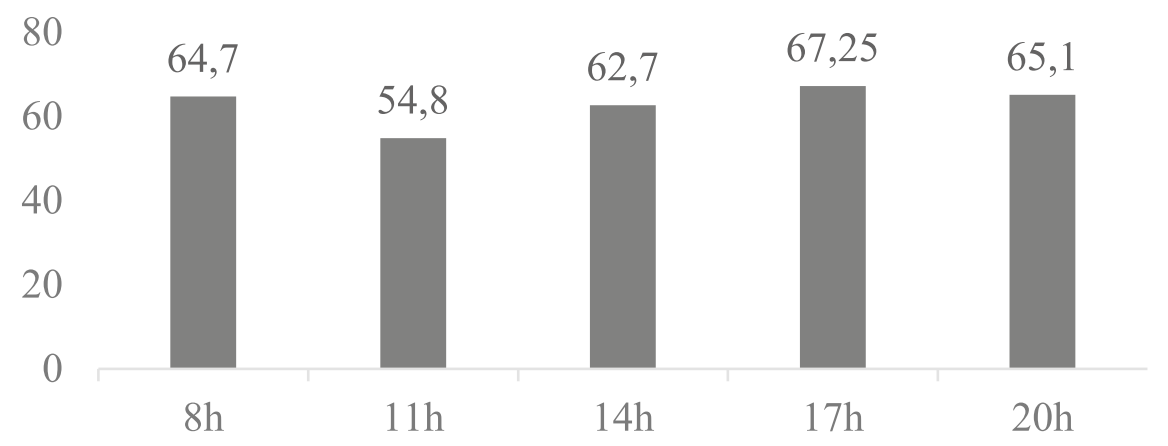

P-VALOR: 0,0158.

$1 \mathrm{Y}=73,890952-2,258333+0,096429 \times 2, \mathrm{R} 2=30,33 \% \quad$ (Eq. 3$)$ 
Siqueira, M. T. S. et al.

A fim de se avaliar a presença de sobrecarga hepática dos animais verificou-se as concentrações de enzimas hepáticas durante o experimento. No entanto, não foi observada diferença entre grupos de borregas avaliadas (tabela 6). A fosfatase alcalina é uma enzima sintetizada em vários tecidos, sendo as maiores concentrações no intestino, rins, ossos e fígado (Kaneko et al.,
2008). Aumento nos níveis de fosfatase alcalina no plasma sanguíneo indicam diversas condições patológicas, como a sobrecarga hepática. De forma geral, os valores dessa enzima estiveram dentro da faixa de referência (Silva et al., 2020), indicando bom funcionamento hepático pelos animais.

Tabela 6 - Concentração média das enzimas hepáticas de borregas alimentadas ou não com leveduras na dieta

\begin{tabular}{lccc}
\hline Tratamento & $\begin{array}{c}\text { Fosfatase Alcalina } \\
\left(\mathbf{U ~ L ~}^{-1}\right)\end{array}$ & AST (U L \\
& 128,06 & GGT $\left(\mathbf{U ~ L}^{-1}\right)$ \\
\hline Controle & 138,73 & 199,33 & 79,80 \\
Rúmen Yeast $^{\circledR}$ & 156,40 & 123,95 & 46,60 \\
Active Flora $^{\circledR}$ & 183,80 & 195,55 & 63,46 \\
Milk Sacc X & & 229,53 & 59,60 \\
P Valor & 0,7386 & 0,5862 & 0,1070 \\
MG & 151,75 & 187,09 & 62,36 \\
CV & 35,13 & 35,64 & 31,73 \\
VR* & $49,00-826,90$ & $41,00-298,00$ & $25,00-146,00$ \\
\hline
\end{tabular}

AST: aspartato aminotransferase; GGT: gamaglutamiltransferase; MG: média geral; CV: coeficiente de variação; VR*: valor de referência para ovinos segundo Silva et al. (2020).

A aspartato aminotransferase (AST) é uma enzima citoplasmática e mitocondrial, presente em vários tecidos como fígado, músculos esquelético e cardíaco. Essa enzima quando identificada acima das concentrações consideradas normais, indica que o animal pode desenvolver lesão hepato-celular secundária, oriundo da excessiva mobilização lipídica. As concentrações de AST estiveram dentro do intervalo considerado normal para a espécie (Silva et al., 2018), indicando mais uma vez que esses animais não desenvolveram lesão hepato-celular.

A gamaglutamiltransferase (GGT) deve ser levada em consideração juntamente com a AST, já que ambas podem indicar se há ou não ocorrência de injúrias ao tecido hepático. Os níveis de GGT podem apresentar aumento imediato caso haja lesão hepática aguda, pois pode ocorrer liberação de fragmentos da membrana que contenham a enzima (Paula, 2015). Em ambos os tratamentos essa enzima está dentro dos valores de referência descritos por Silva et al. (2020), indicando que o fígado desses animais se encontrava em um metabolismo adequado.

Os metabólitos energéticos são eficientes em avaliar o status energético do animal, no entanto avaliam de forma indireta o status proteico dos mesmos, fazendo-se necessária a avaliação de alguns metabólitos proteicos, para resultados mais precisos. Deste modo, não houve efeito $(\mathrm{P}<0,05)$ da utilização de leveduras associadas ao mix de enzimas sobre a concentração dos metabólitos proteicos (Tabela 7).

Tabela 7 - Concentração média dos metabólitos de borregas alimentadas ou não com leveduras na dieta

\begin{tabular}{cccccc}
\hline Tratamento & $\begin{array}{c}\text { Proteínas Totais } \\
\left(\mathbf{g ~ d L}^{-1}\right)\end{array}$ & $\begin{array}{c}\text { Ureia } \\
\left(\mathbf{m g ~ d L}^{-1}\right)\end{array}$ & $\begin{array}{c}\text { Ácido Úrico } \\
\left(\mathbf{m g ~ d L} \mathbf{~ d L}^{-1}\right)\end{array}$ & $\begin{array}{c}\text { Albumina } \\
\left(\mathbf{g ~ d L}^{-1}\right)\end{array}$ & $\begin{array}{c}\text { Creatinina } \\
\left(\mathbf{m g ~ d L}^{-1}\right)\end{array}$ \\
\hline Controle & 4,26 & 88,79 & 0,05 & 4,14 & 0,86 \\
Rúmen Yeast $^{\circledR}$ & 4,93 & 89,46 & 0,10 & 4,14 & 0,80 \\
Active Flora $^{\circledR}$ & 4,55 & 85,99 & 0,10 & 4,06 & 0,80 \\
Milk Sacc X $^{\circledR}$ & 4,52 & 92,06 & 0,07 & 3,95 & 0,79 \\
P Valor & 0,1373 & 0,7000 & 0,5078 & 0,8670 & 0,7395 \\
MG & 4,57 & 89,08 & 0,08 & 4,07 & 0,81 \\
CV & 9,17 & 9,03 & 30,16 & 10,04 & 12,87
\end{tabular}


Avaliação dos parâmetros nutricionais e metabólicos de borregas alimentadas com leveduras na ração

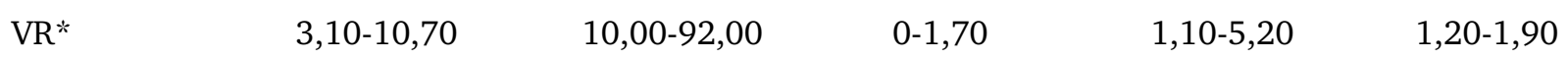

MG: média geral; CV: coeficiente de variação; VR*: valor de referência para ovinos segundo Silva et al. (2020).

Não houve diferença estatística entre os tratamentos para proteínas totais e albumina, que mantiveram seus valores dentro do esperado (Silva et al., 2020). Ambos metabólitos demonstram, o estado nutricional proteico, sendo considerados os indicadores mais sensíveis para determina-lo, já que valores baixos indicam inadequado consumo proteico (Oliveira et al., 2014).

A ureia não apresentou diferença estatística para o presente experimento $(P>0,05)$. Parte da proteína que chega ao rúmen é transformada em amônia, para que possa ser utilizada pela microbiota ruminal na produção de proteína microbiana. Quando há falta de carboidratos na dieta para a completa utilização desta amônia, ela é absorvida pela parede ruminal e levada ao fígado onde é transformada em ureia com alto gasto energético. Essa ureia pode ser eliminada na urina, retornar ao rúmen via saliva ou difusão na parede ruminal e ser eliminada no leite em caso de animal em lactação (Kaneko et al., 2008). Deste modo, é possível que tenha havido uma melhora do sinergismo com o concentrado que era fornecido em maior quantidade na dieta (relação concentrado:volumoso, 70:30). Com isso há maior passagem de nitrogênio, diminuindo a quantidade de nitrogênio livre no rúmen que escaparia e seria metabolizada em ureia no fígado.

O ácido úrico representa de forma indireta crescimento de microrganismos no rúmen uma vez que é utilizado pelos microrganismos ruminais, após a transformação em amônia, como fator de crescimento microbiano, sendo utilizado para a síntese de proteína microbiana, tornando-se assim disponível para o ruminante (Paula, 2015). No presente estudo, foi possível observar que os valores se mostraram equilibrados, não apresentando diferença estatística entre tratamentos.
Os valores de creatinina ficaram abaixo da faixa normal de acordo com Silva et al. (2020) e não houve diferença estatística entre tratamentos, o que pode ter sido causado devido ao fato dos animais estarem confinados, o que resulta em baixo consumo de energia pelo músculo, uma vez que a creatinina tem estreita relação com a massa muscular que varia de acordo com grau de exercício realizado pelos animais.

Pôde-se observar que todos os metabólitos e enzimas hepáticas ficaram dentro dos valores indicados como ideais na literatura. Demonstrando que as dietas utilizadas foram eficientes em manter os metabólitos em níveis adequados, mantendo os animais livres de sobrecarga hepática e renal. Tal fato ocorreu devido ao sinergismo entre as enzimas e leveduras, que contribuíram para uma adequada nutrição dos animais, mantendo o organismo dos mesmos sem alterações significativas.

\section{Conclusão}

A inclusão de leveduras vivas ou inativadas na dieta para borregas não influencia na digestibilidade da dieta, no consumo de matéria seca dos animais e também não altera o perfil metabólico dos mesmos, podendo ser utilizadas em dietas com elevada proporção de grãos e mix de enzimas exógenas.

\section{Aprovação do Comitê de Ética}

O protocolo experimental deste trabalho foi aprovado pela Comissão de Ética na Utilização de Animais (CEUA) da Universidade Federal de Uberlândia sob o número 092/17.

\section{Referências}

Aguiar, S. R.; Ferreira, M. De A.; Batista, A. M. V.; Carvalho, F. F. R. De; Bispo, S. V.; Monteiro, P. de B. S. 2007. Desempenho de ovinos em confinamento alimentados com níveis crescentes de levedura e ureia. Acta Scientiarum. Animal Sciences, 29: 411-416. Doi: 10.4025/ actascianimsci.v29i4.1007.

Carvalho, M. B. 2008. Semiologia do Sistema Urinário. 389-409. In: Feitosa, F.L. Semiologia Veterinária: a arte do diagnóstico. São Paulo.

Cruz, B. C. C. da; Portela, C. F; Cruz, C. L. dos S.; Bittencourt, D.; Cruz, C. A. C. da. 2010. Metabolismo Ruminal: pH, N-NH3, crescimento e eficiência microbiana. PUBVET, 4: 1-15.

Friedewald, W. T.; Levv, R. I.; Fredrickson, D. S. 1972. Estimation of the concentration of low-density lipoprotein in plasma, without use of the preparative ultracentrifuge. Clinical Chemistry. 18, 499-502. Disponivel em: http://clinchem.aaccjnls.org/content/clinchem/18/6/499.full.pdf.

Gomes S. P.; Borges I.; Borges A. L. C. C.; Macedo Junior G. L.; Campos W. E.; Brito T. S. 2012. Tamanho de partícula do volumoso e freqüência de alimentação sobre o metabolismo energético e protéico em ovinos, considerando dietas com elevada participação de concentrado. Revista Brasileira de Saúde e Produção Animal, 13, 732-744. Disponível em: http://www.rbspa.ufba.br/index.php/rbspa/article/viewArticle/2340.

Gouveia, L. N. F.; Maciel, M. V.; Soares, P. C.; Silva Neto, I. F.; Gonçalves, D. N. A.; Batista, A. M. V.; Carvalho, F. F. R. 2015. Perfil metabólico de ovinos em crescimento alimentados com dietas constituídas de feno ou silagem de maniçoba e palma forrageira. Pesquisa Veterinária Brasileira, 35, 5-9.

Igarasi, M. S.; Arrigoni, M. B.; Souza, A. A.; Silveira, A. C.; Martins, C. L.; Oliveira, H. N. 2008. Desempenho de bovinos jovens alimentados com dietas contendo grão úmido de milho ou sorgo. Revista Brasileira de Zootecnia, 37: 513-519. https://doi.org/10.1590/S151635982008000300017 .

Kaneko, J. J., Harvey, J. W., Bruss, M. L. 2008. (Eds.) Clinical Biochemistry of Domestic Animals. San Diego, Academic Press. 
Siqueira, M. T. S. et al.

Kozloski, G. V. 2011. Bioquímica dos Ruminantes, 3oe ed. Revista Ampliada, editora UFSM.

Kruskal, W. H., Wallis, W. A. 1952. Use of ranks in one-criterion variance analysis. Journal American Statistical Association, 47, 583-621.

Levene, H. 1960. Robust Test for Equality of Variances, in I. O. et al., ed., 'Contributions to Probability and Statistics: Essays in Honor of Harold Hotteling', Stanford University Press, California, United States, 278-292.

Maynard, L. A.; Loosli, J. K.; Hintz, H. F.; Warner, R. G. 1984. Nutrição animal. 3. ed. Rio de Janeiro: F. Bastos.

Nacional Research Council - NRC. 2007. Nutrient requirements of small ruminants: Sheep, goats, cervids, and new world camelids. Washington, D. C.: National Academy Press.

Neumann, M.; Ost, P. R.; Pellegrini, L. G. de; Mello, S. E. G. de; Silva, M. A. A. da; Nornberg, J. L. 2008. Utilização de leveduras vivas (Saccharomyces cerevisiae) visando à produção de cordeiros Ile de France superprecoces em sistema de creep-feeding. Ciência rural, 38: 2285-2292.

Oliveira, R. P. M.; Maduro, A. H. P.; Lima, E. S.; Oliveira, F. F. 2014. Perfil metabólico de ovelhas Santa Inês em diferentes fases de gestação criadas em sistema semi-intensivo no Estado do Amazonas. Ciência Animal Brasileira, 15, 81-86.

Reece, W. O. 2006. Função Renal nos Mamíferos. 68-96. In: REECE, W. O. DUKES - Fisiologia dos animais domésticos. 12. ed. Rio de Janeiro, Guanabara Koogan.

Santos I. A., Nogueira L. A. H. 2012. Estudo energético do esterco bovino: seu valor de substituição e impacto da biodigestão anaeróbia. Revista Agrogeoambiental, 4, 1. Disponível em: https://agrogeoambiental. ifsuldeminas.edu.br/index.php/Agrogeoambiental/article/view/373. DOI: http://dx.doi.org/10.18406/2316-1817v4n12012373.
Santos, R. P.; Sousa, L. F.; Sousa, J. T. L.; Andrade, M. E. B.; Macedo Júnior, G. L.; Silva, S. P. 2015. Parâmetros sanguíneos de cordeiros em crescimento filhos de ovelhas suplementadas com níveis crescentes de propilenoglicol. Revista Brasileira de Ciências Agrárias, 10, 473-478.

Shapiro S. S., Wilk M. B. 1965. An Analysis of Variance Test for Normality, (Complete Samples). Biometrika. 52(3/4), 591-611. Disponível em: https://dx.doi.org/10.2307/2333709.

Silva, D. A. P.; Varanis, L. F. M.; Oliveira, K. A.; Sousa, L. M.; Siqueira, M. T. S.; Macedo Júnior, G. L. 2020. Parâmetros de metabólitos bioquímicos em ovinos criados no Brasil. Caderno de Ciências Agrárias, 12, 01-08. DOI: https://doi.org/10.35699/2447-6218.2020.20404.

Tavares, L.; Paula Schmidt, A.; Gonçalves Malaguez, E.; Piagetti Noschang, J.; Cassal Brauner, C.; Nunes Corrêa, M. 2020. Utilização de Saccharomyces cerevisiae: relação com consumo e GMD em trocas de dieta em ovinos. Anais do Salão Internacional de Ensino, Pesquisa e Extensão, 10: 1-6.

Van Cleef E. H. C. B., Ezequiel J. M. B., D’aurea A. P., Fávaro, V. R., Sancanari, J. B. D. 2014. Crude glycerin in diets for feedlot Nellore cattle. Revista Brasileirade Zootecnia, Viçosa, 43, 2, 8691. Disponível em: http://www.scielo.br/scielo.php?pid=S151635982014000200006\&script=sci arttext\&tlng=pt. DOI: http://dx.doi. org/10.1590/S1516-35982014000200006.

Vieira, L. S. 2008. Métodos alternativos de controle de nematóides gastrintestinais em caprinos e ovinos. Embrapa Caprinos e Ovinos - Artigo em periódico indexado. Disponível em: https:// www.alice.cnptia.embrapa.br/bitstream/doc/533824/1/ APIMetodosalternativosdecontroledenematoides.pdf.

Villa, N. A.; Pulgarín, E.F.; Tabares, P. A.; Angarita, E.; Ceballos, A. 2019. Medidas corporales y concentración sérica y folicular de lípidos y glucosa en vacas Brahman fértiles y subfértiles. Pesquisa Agropecuária Brasileira, 44, 1198-1204. Disponível em: https://www.scielo.br/scielo. php? script $=$ sci_abstract\&pid $=$ S0100-204X2009000900019\&lng $=$ pt \& $\mathrm{nrm}=$ iso\&tlng $=$ es. 